\title{
Evaluation of quality of anesthesia and analgesia and of vital signs after intramuscular administration of a combination of butorphanol, medetomidine and alfaxalone in cats
}

\author{
Ye-Won $\mathrm{KIM}^{1)}$, Sang-Il SUH${ }^{1)}$, Ran $\mathrm{CHOI}^{1)}$ and Changbaig $\mathrm{HYUN}^{1) *}$ \\ ${ }^{1)}$ Section of Small Animal Internal Medicine, College of Veterinary Medicine, Kangwon National University, Chuncheon, Republic of \\ Korea
}

(Received 13 May 2015/Accepted 13 October 2015/Published online in J-STAGE 9 November 2015)

\begin{abstract}
This study evaluated the quality of anesthesia, duration of analgesia and changes in vital signs after intramuscular administration of a combination of butorphanol, medetomidine and alfaxalone in domestic cats. Ten healthy adult domestic cats (weighing $2.9 \pm 0.5 \mathrm{~kg}$ ) were used in this study. Rectal temperature $(\mathrm{T})$, pulse rate $(\mathrm{PR})$, respiratory rate $\left(f_{\mathrm{R}}\right)$ and systolic arterial pressure (SAP) were measured and recorded prior to intramuscular (IM) administration of butorphanol $(0.2 \mathrm{mg} / \mathrm{kg})$, medetomidine $(20 \mathrm{ug} / \mathrm{kg})$ and alfaxalone (5 mg/kg) and then every 10 min until return of consciousness. Qualitative scores for induction of anesthesia and recovery were allocated, duration of anesthesia and recovery were calculated, and adverse events were recorded. A needle prick with a 22-gauge hypodermic needle was used to assess analgesia. Scores for induction and recovery quality were acceptable. No significant adverse events except nausea (7/10) and vomiting $(5 / 10)$ were observed. The mean \pm SD times from induction to extubation and to standing (full recovery) were $114 \pm 8$ and $125 \pm 7$ min, respectively. There were statistically significant changes in PR, $f_{\mathrm{R}}$ and SAP after induction of anesthesia. The combination of butorphanol, medetomidine and alfaxalone provided acceptable quality of anesthesia and analgesia and exerted minimal cardiopulmonary effects in domestic cats.
\end{abstract}

KEY WORDS: alfaxalone, anesthesia, butorphanol, domestic cat, medetomidine

doi: 10.1292/jvms.15-0288; J. Vet. Med. Sci. 78(3): 431-433, 2016

Total intravenous anesthesia (TIVA) with a ketamine combination and total intramuscular anesthesia (TIMA) with tiletamine/zolazepam and medetomidine are major anesthetic protocols for dogs and cats in Korea. Alfaxalone

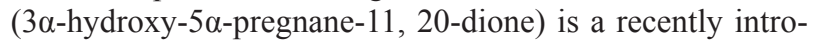
duced induction agent and has been administered intravenously and intramuscularly in cats $[1,2]$. Unlike propofol, alfaxalone minimally affects the cardiorespiratory system at clinical dose rates and can be used with various types of premedication [7]. However, only a few studies have been conducted to evaluate IM use of alfaxalone in cats. Therefore, this study evaluated the quality of anesthesia and analgesia and the changes in vital signs after IM administration of a combination of butorphanol, medetomidine and alfaxalone in cats.

Approval of the animal ethics committee of Kangwon National University was obtained for this experiment prior to the commencement of the study. Ten adult domestic short hair cats (five males, five females; mean body weight, 2.9 $\pm 0.5 \mathrm{~kg}$; body condition score, $2.7 \pm 0.3 / 5$; and age, $1.6 \pm$ $0.5 \mathrm{yrs}$ ) from a cat shelter were used for this study. All cats

*Correspondence to: Hyun, C., Section of Small Animal Internal Medicine, College of Veterinary Medicine, Kangwon National University, Kangwon National University Road 1, Chuncheon 200-701, Korea. e-mail: hyun5188@kangwon.ac.kr

(C)2016 The Japanese Society of Veterinary Science

This is an open-access article distributed under the terms of the Creative Commons Attribution Non-Commercial No Derivatives (by-nc-nd) License $<$ http://creativecommons.org/licenses/by-nc-nd/4.0/>. were healthy based upon physical examination, evaluation of an electrocardiogram (ECG) and serum chemistry and hematologic analyses. Cats were administered butorphanol (0.2 mg/kg; Jaeil Pharmaceutical, Seoul, Republic of Korea), medetomidine (20 $\mu \mathrm{g} / \mathrm{kg}$; Pfizer, Seoul, Republic of Korea) and alfaxalone ( $5 \mathrm{mg} / \mathrm{kg}$; Jurox, Rutherford, Australia) in a single syringe into the dorsal lumbar muscle. The tracheas of all cats were intubated, and the cats were allowed to breathe room air until extubation. Cats were placed on a table covered with a warm blanket.

Times from IM injection to lateral recumbency, orotracheal intubation, sternal recumbency and standing were recorded. Recovery from anesthesia was indicated by the ability to maintain a sternal standing. Duration of anesthesia was calculated as the time from lateral recumbency to extubation. Duration of recovery was calculated as the time from extubation to sternal recumbency. Total procedure time was calculated as the time from IM injection to standing. Qualities of anesthetic induction and recovery were scored using a standardized scale previously described [8]. Evidences of adverse events noted throughout induction and recovery were recorded, including abnormal muscle movements and ECG alteration. Pulse rate (PR), respiratory rate $\left(f_{\mathrm{R}}\right)$, arterial systolic aortic pressure (SAP) and rectal temperature $(\mathrm{T})$ were recorded before administration of medications (T0) and then every 10 min until extubation. The SAP was measured by Doppler flow detector (811-B, Parks Medical Electronics, Aloha, OR, U.S.A.). PR and $f_{\mathrm{R}}$ were obtained manually. The $\mathrm{SpO}_{2}$ was recorded but not considered accurate, and most of the time, thus its values were not included in the results. Rec- 
Table 1. Cardiopulmonary and temperature measurements before (T0) and after intramuscular administration of $0.2 \mathrm{mg} \mathrm{kg}^{-1}$ of butorphanol, $20 \mu \mathrm{g} \mathrm{kg}^{-1}$ of medetomidine and $5.0 \mathrm{mg} \mathrm{kg}^{-1}$ of alfaxalone in cats

\begin{tabular}{|c|c|c|c|c|c|c|}
\hline \multirow{2}{*}{ Variable } & \multicolumn{6}{|c|}{ Time points } \\
\hline & T0 & T10 & $\mathrm{T} 20$ & T30 & $\mathrm{T} 40$ & T50 \\
\hline PR (beats/min) & $156 \pm 17$ & $136 \pm 17$ & $112 \pm 17^{*}$ & $110 \pm 10^{*}$ & $105 \pm 12 *$ & $93 \pm 15^{*}$ \\
\hline $\mathrm{SAP}(\mathrm{mmHg})$ & $141 \pm 11$ & $133 \pm 12$ & $117 \pm 19^{*}$ & $113 \pm 12 *$ & $115 \pm 12 *$ & $107 \pm 12 *$ \\
\hline $\mathrm{T}\left({ }^{\circ} \mathrm{C}\right)$ & $38.9 \pm 0.7$ & $38.3 \pm 0.6$ & $37.9 \pm 0.7$ & $37.9 \pm 0.7$ & $37.8 \pm 0.8$ & $37.4 \pm 0.6$ \\
\hline$f \mathrm{R}$ (breaths/min) & $36 \pm 11$ & $32 \pm 11$ & $29 \pm 7 *$ & $27 \pm 5^{*}$ & $26 \pm 7^{*}$ & $26 \pm 8^{*}$ \\
\hline \multirow{2}{*}{ Variable } & \multicolumn{6}{|c|}{ Time points } \\
\hline & T60 & $\mathrm{T} 70$ & T80 & T90 & $\mathrm{T} 100^{\$}$ & $\mathrm{~T} 110^{\#}$ \\
\hline PR (beats/min) & $86 \pm 17 *$ & $97 \pm 21^{*}$ & $108 \pm 11^{*}$ & $119 \pm 23^{*}$ & $136 \pm 9$ & $132 \pm 15$ \\
\hline $\mathrm{SAP}(\mathrm{mmHg})$ & $108 \pm 13^{*}$ & $105 \pm 12 *$ & $102 \pm 19 *$ & $115 \pm 11^{*}$ & $125 \pm 14^{*}$ & $132 \pm 12$ \\
\hline $\mathrm{T}\left({ }^{\circ} \mathrm{C}\right)$ & $37.9 \pm 0.3$ & $38.2 \pm 0.2$ & $37.8 \pm 0.3$ & $37.6 \pm 0.5$ & $37.8 \pm 0.4$ & $38.0 \pm 0.5$ \\
\hline$f \mathrm{R}$ (breaths/min) & $26 \pm 3^{*}$ & $27 \pm 11 *$ & $25 \pm 7^{*}$ & $24 \pm 4^{*}$ & $30 \pm 9$ & $31 \pm 11$ \\
\hline
\end{tabular}

Mean \pm SD. PR, pulse rate; SAP, systolic arterial pressure; T, rectal temperature; $f_{\mathrm{R}}$, respiratory rate. ${ }^{\$} \mathrm{~N}=9$, ${ }^{\#} \mathrm{~N}=7$. *Significant difference from baseline (T0).

tal temperature was measured using a rectal probe (VSM7; Votem, Chuncheon, Republic of Korea). Continuous lead II ECG monitoring (VH-1, CU Medical-Systems, Wonju, Republic of Korea) was performed from induction to recovery. A needle prick with a 22-gauge hypodermic needle was used to assess analgesia as described previously [3]. Duration of analgesia was defined as the time from initiation of needle pricking to the time of a positive response.

The statistical software used in statistical analysis was SPSS 15.0 for Windows (SPSS Inc., Chicago, IL, U.S.A.). Normal distribution was confirmed by KolmogorovSmirnov test. One-way ANOVA with repeated measures was performed with the same parameters between baseline and post-induction values with Dunnett's test for post hoc analysis. Significance was set at $P<0.05$.

The induction quality and recovery quality scores in this study population were $1(0-3)$ and $1(0-3)$, respectively. Five of the 10 cats were mildly ataxic during recovery. Adverse effects including nausea (7/10) and vomiting (5/10) were found in our study population during the induction of anesthesia. Although no morphological alterations in ECG were detected, sinus bradycardia $(<100$ beats per min; 86 \pm 17 beats per min at T60) was recorded in all cats. No cats showed laryngeal complications (e.g., laryngeal spasms) during intubation or recovery, even though we did not desensitize the larynx with lidocaine. The mean \pm SD times from IM injection to lateral recumbency and orotracheal intubation were $11 \pm 7$ and $15 \pm 6 \mathrm{~min}$, respectively. The mean \pm SD times from induction to extubation and to standing were $114 \pm 8$ and $125 \pm 7 \mathrm{~min}$, respectively. The mean duration of analgesia (based on positive response in the needle prick test) was $55 \pm 17 \mathrm{~min}$.

There were statistically significant changes in PR, $f_{\mathrm{R}}$ and SAP after administration of butorphanol, medetomidine and alfaxalone (Table 1). The PR and SAP were gradually lower after induction of anesthesia. The $f_{\mathrm{R}}$ was also significantly lower during anesthesia. Rectal temperature decreased nonsignificantly with time.

Grubb et al. also found poor quality anesthesia and un- predictable induction time in cats using a combination of alfaxalone $(5 \mathrm{mg} / \mathrm{kg})$ with dexmedetomidine $(0.01 \mathrm{mg} / \mathrm{kg})$ and hydromorphone $(0.1 \mathrm{mg} / \mathrm{kg})$ by IM injection [2]. As noticed by Grubb et al. [2], there was large individual variation in time to reach complete induction (5-23 $\mathrm{min}$ ) among the cats used in our study. In this study, intubation of the trachea in half of the cats $(5 / 10)$ was not easy. It took 5 min to complete endotracheal intubation for some cats. However, intubation in cats is generally tougher than in dogs, especially if the trachea is not desensitized. Although intubation was done by an experienced vet (Choi), it was not easy to intubate half of the cats, because we did not desensitize the trachea during intubation. We were afraid that desensitization might affect the quality of intubation and might lead us to underestimate the quality of intubation.

The depth of analgesia varied from light to deep among cats. The time to reach a perceivable depth of analgesia was unpredictable (11-32 $\mathrm{min})$, although the time to full recovery or standing was almost $2 \mathrm{hr}$ in the majority of cats. However, this anesthesia protocol was sufficient enough for dental scaling and non-painful procedures, such as grooming or diagnostic imaging studies (unpublished data). Unlike the previous study by Grubb et al [2], our study found that most of the cats were calm and that induction and recovery were smooth following IM administration of alfaxalone, although intubation in most cats was not easy. However, the times of induction and recovery from anesthesia were unpredictable and varied individually. Furthermore, the volume of alfaxalone was too excessive for a single IM injection in cats [2]. Analgesia was achievable and persistent for $\sim 1 \mathrm{hr}$.

The PR in this study was reduced after induction and then significantly reduced with time. Profound bradycardia ( $<100$ beats per min) might be due to an alpha-adrenergic effect from medetomidine [4]. Although a high dose of alfaxalone $(50 \mathrm{mg} / \mathrm{kg})$ could reduce PR in cats [6], alfaxalone might not have affected the PR in this study because we only used the recommended dose. As the duration of action of medetomidine in cats is up to $2 \mathrm{hr}$ [5], the prolonged recovery and bradycardia in this study might be attributable to the 
effect of medetomidine. There was a persistent reduction in $f \mathrm{R}$ in this study, although no cats showed apnea (no spontaneous respiration for longer than $1 \mathrm{~min}$ ). Several studies found that alfaxalone could cause dose-related respiratory depression or apnea $[1,6,9]$. However, marked respiratory suppression including apnea was not observed in this study. The arterial blood pressure was significantly lowered during anesthesia in this study, although no cats showed clinically significant hypotension ( $\mathrm{SAP}<80-90 \mathrm{mmHg}$ ) requiring immediate medical intervention. Muir et al. reported dosedependent cardiovascular depression caused by alfaxalone injection in unpremedicated cats at the supraclinical dose of $50 \mathrm{mg} / \mathrm{kg}$ (10 times higher than the dose used in this study) [6]. The profound bradycardia caused by medetomidine might have contributed to the lowering of the arterial blood pressure in this study, despite the vasoconstriction from the alpha-adrenergic effect. Beths et al. found that the body temperature of cats administered alfaxalone was significantly lowered [1]. However, in this study, the body temperature was well maintained in most of the cats. This is probably because all the cats were lying on a warm blanket.

In conclusion, this study evaluated the quality of anesthesia, duration of analgesia and changes in vital signs after IM administration of a combination of butorphanol, medetomidine and alfaxalone in cats. There were significant reductions in $f \mathrm{R}, \mathrm{PR}$ and SAP during anesthesia, although the reductions were clinically insignificant. Although the study population was limited to a small number of healthy young cats and not capable of obtaining sufficient statistical power to verify our study results, this anesthetic protocol provided acceptable quality of anesthesia and analgesia and exerted minimal cardiopulmonary effects in domestic cats.

\section{REFERENCES}

1. Beths, T., Touzot-Jourde, G., Musk, G. and Pasloske, K. 2014. Clinical evaluation of alfaxalone to induce and maintain anaesthesia in cats undergoing neutering procedures. J. Feline Med. Surg. 16: 609-615. [Medline] [CrossRef]

2. Grubb, T. L., Greene, S. A. and Perez, T. E. 2013. Cardiovascular and respiratory effects, and quality of anesthesia produced by alfaxalone administered intramuscularly to cats sedated with dexmedetomidine and hydromorphone. J. Feline Med. Surg. 15: 858-865. [Medline] [CrossRef]

3. Ko, J. C., Abbo, L. A., Weil, A. B., Johnson, B. M. and Payton, M. A. 2007. A comparison of anesthetic and cardiorespiratory effects of tiletamine-zolazepam-butorphanol and tiletaminezolazepam-butorphanol-medetomidine in cats. Vet. Ther. 8: 164-176. [Medline]

4. Lamont, L. A., Bulmer, B. J. and Grimm, K. A. 2001. Cardiopulmonary evaluation of the use of medetomidine hydrochloride in cats. Am. J. Vet. Res. 62: 1745-1749. [Medline] [CrossRef]

5. Lemke, K. A. 2007. Anticholinergics and sedatives. pp. 203-240. In: Lumb and Jones' veterinary anesthesia, 4th ed. (Tranquilli, W. J., Thurmon J. C. and Grimm, K. A. eds.), Blackwell, Ames.

6. Muir, W., Lerche, P., Wiese, A., Nelson, L., Pasloske, K. and Whittem, T. 2009. The cardiorespiratory and anesthetic effects of clinical and supraclinical doses of alfaxalone in cats. Vet. Anaesth. Analg. 36: 42-54. [Medline] [CrossRef]

7. Pasloske, K., Gazzard, B., Perkins, N., Dunlop, C. and Whittem, T. 2005. A multicentre clinical trial evaluating the efficacy and safety of Alfaxan administered to dogs for induction and maintenance of anaesthesia. p. 556. Proceedings of the 48th Annual BSAVA Congress, Birmingham, U.K.

8. Sams, L., Braun, C., Allman, D. and Hofmeister, E. 2008. A comparison of the effects of propofol and etomidate on the induction of anesthesia and on cardiopulmonary parameters in dogs. Vet. Anaesth. Analg. 35: 488-494. [Medline] [CrossRef]

9. Whittem, T., Pasloske, K. S., Heit, M. C. and Ranasinghe, M. G. 2008. The pharmacokinetics and pharmacodynamics of alfaxalone in cats after single and multiple intravenous administration of Alfaxan ${ }^{\circledR}$ at clinical and supraclinical doses. J. Vet. Pharmacol. Ther. 31: 571-579. [Medline] [CrossRef]

\section{Appendix 1. Quality of anesthetic induction and recovery scores used in this study Induction scores: 0 , smooth uncomplicated; 1 , uncomplicated; 2 , induction difficulty; and 3, induction rough. Recovery score: 0 , perfect, walking without ataxia, smooth uncomplicated; 1 , good, walking with minimal ataxia, uncomplicated; 2 , adequate, walking with moderate ataxia, recovery difficult; 3 , rough, walking with significant ataxia or crawling.}

\section{Appendix 2. Needle prick for determining degree of analgesia}

A needle prick with a 22-gauge hypodermic needle was used to assess analgesia. The needle prick analgesic test was standardized in all cats and started from $5 \mathrm{~min}$ after anesthesia. The test was scored as a "yes" or "no" response. When there was a positive response, such as limb withdrawal, skin twitching or any other purposeful movements in reaction to the needle pricking, the response was recorded as "no" analgesia. When there was no response, the response of the animal was considered "yes" for analgesia until the next stimulus was applied. The sequence of areas subjected to needle pricking was as follows: front limb near radial-ulnar area, ventral midline of the abdomen and rear limb near the tibial area. The test was repeated every $10 \mathrm{~min}$ until the cat showed a positive response in all locations. 